\title{
Electronic structure of multilayer allotropes of 2D silicon carbide
}

\author{
A. V. Kalashnikov ${ }^{\dagger}$ A. V. Tuchin, L. A. Bityutskaja \\ †akalash49@gmail.com
}

Voronezh State University, 1 University Sq., Voronezh, 394018, Russia

\begin{abstract}
The transition from bulk material to low-dimensional structures (2D, 1D) is accompanied not only by the appearance of new electrophysical properties, but also by a change in the symmetry of the elementary lattice caused by the violation of longrange order in one or several crystallographic directions. Therefore, of a particular interest is the study of the phenomenon of allotropy in $2 \mathrm{D}$ and quasi-2D crystals. In the present work, on the basis of quantum chemical calculations from first principles, we studied the size-depended allotropy and the structure-dependent properties of low-dimensional silicon carbide ( $\mathrm{SiC}$ ), obtained on the basis of $\mathrm{SiC}$ monolayers with a stoichiometric composition of 1:1. It has been established that 2D allotropes of $\mathrm{SiC}$ form a family of semiconductor structures with a different band structure (both direct-band- and non-direct-bandsemiconductors) and charge properties. A deeper analysis of the geometric and energy parameters made it possible to establish the possibility of the sustainable existence of four topological 2D SiC types, differing in the way and order of alternation of layers, and the allotropic modifications obtained are not characteristic of bulk material. Accounting for the spatial separation of the charge as a result of the formation of a covalent polar bond between the carbon and silicon atoms that made up the structure allowed detecting the formation of an effective charge within the monolayer. After the analysis of the magnitude and sign of the specific surface charge, a correlation was established between this parameter and the geometry of the optimized structure. Thus, taking into account the charge properties of $2 \mathrm{D} \mathrm{SiC}$, it is possible to trace structural changes in the system, identify a specific allotrope, and establish the order in which the monolayers are laid. Characteristic patterns in the charge distribution are a prerequisite for the production of composite materials based on $2 \mathrm{D}$ allotropes of $\mathrm{SiC}$.
\end{abstract}

Keywords: allotropy, low-dimensional materials, covalent compounds, effective charge.

УДК: 541.7/.73

\section{Электронная структура мультислойных аллотропов 2D-карбида кремния}

\author{
Калашников А. В. ${ }^{\dagger}$, Тучин А. В., Битюцкая Л. А. \\ Воронежский государственный университет, Университетская пл., 1, Воронеж, 394018, Россия
}

Переход от объемного материала к структурам пониженной размерности (2D, 1D) сопровождается не только появлением новых электрофизических свойств, но и изменением симметрии элементарной решётки, вызванным нарушением дальнего порядка в одном или нескольких кристаллографических направлениях. Поэтому особый интерес представляет исследование явления политипизма в 2D и квази-2D кристаллах. B настоящей работе на основе квантово-химических расчетов из первых принципов проведено исследование размерного политипизма и структуро-зависимых свойств низкоразмерного карбида кремния ( $\mathrm{SiC})$, полученного на основе монослоев $\mathrm{SiC}$ со стехиометрическим составом 1:1. Установлено, что 2D-аллотропы $\mathrm{SiC}$ образуют семейство полупроводниковых структур с различной зонной структурой (как прямозонные, так и не прямозонные полупроводники) и зарядовыми свойствами. Более глубокий анализ геометрических и энергетических параметров позволил установить возможность устойчивого существования 4 топологических типов 2D $\mathrm{SiC}$, отличающихся способом укладки и порядком чередования слоёв, а полученные аллотропные модификации не характерны для объемного материала. Учёт пространственного разделения заряда в результате формирования ковалентной полярной связи между атомами углерода и кремния, составляющими структуру, позволил обнаружить формирование эффективного заряда в пределах монослоя. В результате анализа величины и знака удельного поверхностного заряда была установлена корреляция данного параметра и геометрии оптимизированной структуры. Таким образом, учет зарядовых свойств 2D SiC позволяет проследить структурные изменения в системе, идентифицировать конкретный аллотроп и установить порядок укладки монослоев. Характерные закономерности в распределении заряда являются предпосылкой для получения композитных материалов на основе 2D аллотропов $\mathrm{SiC}$.

Ключевые слова: аллотропия, низкоразмерные материалы, ковалентные соединения, эффективный заряд. 


\section{1. Введение}

Активное исследование многослойных 2D структур привело к открытию новых материалов с уникальным электрофизическими свойствами [1-4]. На сегодняшний день экспериментально получен широкий спектр стабильных низкоразмерных структур. Среди которых можно выделить три класса материалов: со слабой межслоевой связью, обусловленной Ван-дер-Ваальсовым типом взаимодействия (графен, силицен, станен и т.д.); со средне-сильной межслоевой связью, обусловленной наличием, наряду с Ван-дер-Ваальсовым, ионного механизма; слоистые структуры с ковалентным типом межслоевого взаимодействия [5-16].

В последние десятилетие особое внимание уделялось структурам первого типа, что связано с относительной простотой получения таких материалов (механическое расслоение), между тем, наличие отработанных, с высокой степенью воспроизводимости, методов получения атомарно тонких пленок материалов с ковалентным типом межслоевого взаимодействия и привлекательность электронных свойств активизировали интерес к всестороннему исследованию таких структур. В данной области все больше усилий направлено на прогнозирование структуры и функциональных свойств новых 2D материалов, таких, как монослои бора (hB) с малым уровнем деформации слоя, монослои карбида бора (BC), силицида бора (BSi), карбида алюминия (AlC), карбида кремния ( $\mathrm{SiC})$, нитрида углерода $(\mathrm{CN})$, германена, тетрагонального карбида титана (TiC), карбида олова $(\mathrm{SnC})$ и других соединений элементов 3-4 групп [10-16].

Среди низкоразмерных структур с ковалентным типом межслоевого взаимодействия особый интерес представляет $\mathrm{SiC}$, объемный прекурсор которого обладает широким спектром политипных модификаций (известно порядка 200 политипов $\mathrm{SiC}$ ), что, в случае структур пониженной размерности, означает наличие богатых электрофизических свойств [17-31]. Поэтому задача исследования структурной стабильности исвойств различных топологических форм 2D фаз карбида кремния в настоящее время является актуальной. Между тем, не смотря на большое число работ по исследованию структурно-зависимых свойств многослойного $\mathrm{SiC}$ [25-29], вопрос размерного политипизма, всё ещё остается мало изученным.

Поэтому, целью настоящей работы является исследование структурной эволюции аллотропных модификаций 2D карбида кремния на основе квантово-химических расчетов из первых принципов.

\section{2. Используемые подходы и методы исследования}

Исследование электронной структуры 2D аллотропов карбида кремния проводилось теорией функционала электронной плотности (DFT) в приближении локальной спиновой плотности (LSDA) [32,33] c использованием валентно-расщепленного базиса 3-21G и числом k-точек 80000. Данный метод широ- ко используется для моделирования многослойных 2D наноматериалов, в том числе мультиграфена [34 - 37]. Расчеты выполнены в Суперкомпьютерном центре Воронежского государственного университета с использованием специализированного программного комплекса для решения квантово-химических задач Gaussian09.

Для исследования распределения зарядов и их корреляции со структурой и свойствами $2 \mathrm{D} \mathrm{SiC}$, после проведения процесса оптимизации геометрии структур, были рассчитаны следующие характеристики (Табл. 1):

- внутрислойные длины связей между атомами кремния и углерода $\left(R_{\mathrm{Si}-\mathrm{C}}\right)$ (Рис. 1$)$;

- межслоевые расстояния $R_{\mathrm{sh}}=Z_{\mathrm{Si} / \mathrm{C}-\mathrm{SH} 1}-Z_{\mathrm{Si} / \mathrm{C}-\mathrm{SH} 2}$, где $Z_{\mathrm{Si} / \mathrm{C}}-$ координаты кремния/углерода по оси $z$ (исходная топология структуры ориентирована таким образом, что вектора трансляции $\mathbf{a}$ и $\mathbf{b}$ находятся в плоскости $X Y$ (Рис. 1));

- приведенная энергия связи $E_{b}=\left(E_{t o t}\left(\mathrm{Si}_{N} \mathrm{C}_{N}\right)-\right.$ $\left.-N E_{\text {tot }}(\mathrm{Si})-N E_{\text {tot }}(\mathrm{C})\right) / 2 N$, где $N-$ число атомов одного сорта в элементарной ячейке, $E_{t o t}\left(\mathrm{Si}_{N} \mathrm{C}_{N}\right)-$ полная энергия системы, $E_{t o t}(\mathrm{Si})-$ полная энергия атомарного кремния, $E_{t o t}(\mathrm{C})$ - полная энергия атомарного углерода;

- межслоевая энергия связи $E_{b s h}=\left(E_{t o t}\left(\mathrm{Si}_{n} \mathrm{C}_{n}\right)-\right.$ $\left.-n E_{t o t}(\mathrm{SiC})\right) / n$, где $E_{t o t}(\mathrm{SiC})-$ полная энергия однослойного аллотропа $\mathrm{SiC}, E_{t o t}\left(\mathrm{Si}_{n} \mathrm{C}_{n}\right)-$ полная энергия системы из $n$ слоёв;

- ширина запрещенной зоны $E_{g}-$ минимальная энергия прямозонного $\left(E_{g(\text { direct })}\right)$ и не прямозонного перехода $\left(E_{g(\text { indirect })}\right)$;

- удельный атомарный заряд $\left(Q_{b}\right)$;

- эффективного удельный поверхностный заряд $Q_{b s h}=\left(\Sigma\left(Q_{b(\mathrm{Si})}\right)+\Sigma\left(Q_{b(\mathrm{C})}\right)\right) / N$, где $Q_{b(\mathrm{C})}, Q_{b(\mathrm{Si})}-$ удельный заряд атомов углерода и кремния слоя, $N$ - число атомов в слое.

Расчет энергетических характеристик основан на результатах квантово-химического моделирования электронной структуры 2D аллотропов $\mathrm{SiC}$. При этом полученные данные соотносятся с экспериментом (для политипа $\mathrm{A} \bar{A} \mathrm{~A}$, в предложенной терминологии, квази-2D SiC: $R_{\mathrm{Si}-\mathrm{C}}=1.78-1.89 \AA, R_{\mathrm{sh}} \sim 3.09 \AA, E_{g} \sim 2.58$ эВ) [20] и теоретическими (например, для монослоя $\mathrm{SiC}$ :

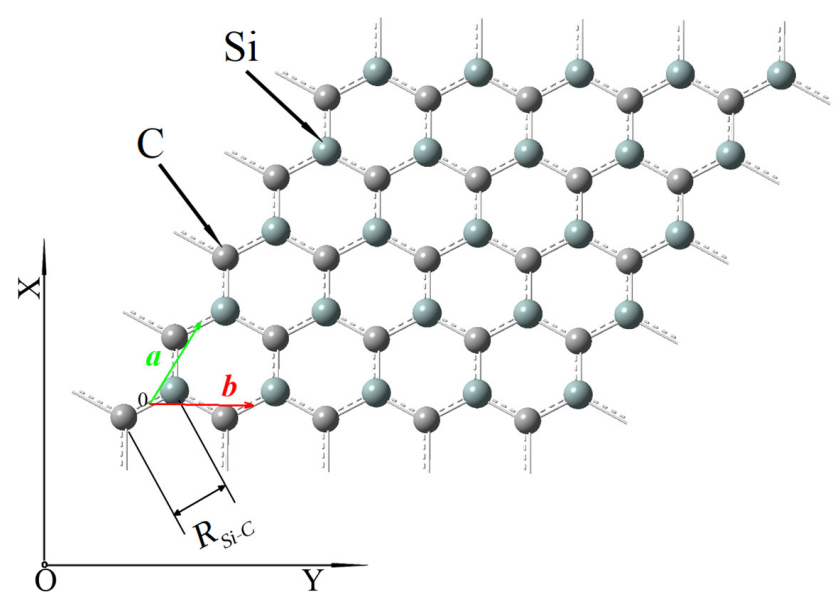

Рис. 1. Макроячейка $5 \times 5$ однослойного $\mathrm{SiC}$.

Fig. 1. Macrocell $5 \times 5$ of single-layer $\mathrm{SiC}$. 
Табл. 1. Электрофизические свойства $2 \mathrm{D}$ аллотропов $\mathrm{SiC}$ в зависимости от числа и конфигурации слоев $\left(R_{\mathrm{Si-C}}-\right.$ длина связи между атомами углерода и кремния в пределах слоя; $R_{s h}-$ межслоевое расстояние, $E_{b}-$ приведенная энергия связи; $E_{b s h}-$ приведенная межплоскостная энергия связи; $E_{g(\text { direct) }}, E_{g(\text { inderect })}-$ ширина запрещенной зоны при прямозонном и непрямозонном переходе; $Q_{b s h}-$ удельный заряд слоя).

Table 1. Electrophysical properties of $2 \mathrm{D} \mathrm{SiC}$ allotropes depending on the number and configuration of layers $\left(R_{\mathrm{Si}-\mathrm{C}}\right.$ is the length of bond between carbon and silicon atoms within the layer; $R_{s h}$ is the interlayer distance; $E_{b}$ is the reduced binding energy; $E_{b s h}$ is the reduced interplanar binding energy; $E_{g(\text { direct) }}, E_{g \text { (inderect) }}$ is the direct and indirect band gap; $Q_{b s h}$ is the specific charge layer).

\begin{tabular}{|c|c|c|c|c|c|c|c|}
\hline $\begin{array}{l}\text { Структура } \\
\text { Structure }\end{array}$ & $R_{\mathrm{Si}-\mathrm{C}}, \AA$ & $R_{s h}, \AA$ & $\begin{array}{l}Q_{b s h}, \text { Кл } \cdot \mathrm{e} \\
Q_{b s h}, \mathrm{C} \cdot \mathrm{e}\end{array}$ & $\begin{array}{l}E_{g(\text { direct })}, \ni \mathrm{B} \\
E_{g(\text { direct })}, \mathrm{eV}\end{array}$ & $\begin{array}{l}E_{g(\text { inderect) })}, \ni \mathrm{B} \\
E_{g \text { (inderect) }}, \mathrm{eV}\end{array}$ & $\begin{array}{l}E_{b}, \ni \mathrm{B} / \text { атом } \\
E_{b}, \mathrm{eV} / \text { atom }\end{array}$ & $\begin{array}{l}E_{b s h}, \text { эВ/ слой } \\
E_{b s h}, \mathrm{eV} / \text { layer }\end{array}$ \\
\hline Графен/Graphene & 1.42 & - & 0 & 0.022 & 0.022 & 10.902 & - \\
\hline $\mathrm{A}$ & 1.805 & - & 0 & 1.998 & 1.998 & 8.322 & - \\
\hline $\mathrm{AA}$ & 1.804 & 3.380 & \pm 0.0125 & 1.445 & 1.366 & 8.399 & 0.154 \\
\hline AAA & 1.804 & 3.190 & -0.014 & 1.134 & 1.134 & 8.434 & 0.224 \\
\hline $\mathrm{A} \overline{\mathrm{A}}$ & 1.846 & 2.200 & 0 & 3.074 & 2.149 & 8.444 & 0.242 \\
\hline $\mathrm{A} \bar{A} \mathrm{~A}$ & 1.837 & 2.334 & +0.077 & 2.175 & 1.568 & 8.524 & 0.403 \\
\hline $\mathrm{AB}$ & 1.805 & 3.243 & \pm 0.001 & 1.339 & 1.341 & 8.401 & 0.156 \\
\hline $\mathrm{ABA}$ & 1.804 & 3.174 & +0.031 & 1.132 & 1.132 & 8.433 & 0.223 \\
\hline $\mathrm{A} \bar{A}^{\prime}$ & 1.805 & 2.673 & 0 & 1.484 & 1.386 & 8.280 & -0.084 \\
\hline $\mathrm{A} \bar{A}^{\prime} \mathrm{A}$ & 1.804 & 3.156 & +0.002 & 1.344 & 1.325 & 8.316 & -0.014 \\
\hline $\mathrm{AA}^{\prime}$ & 1.843 & 2.214 & \pm 0.022 & 3.032 & 2.150 & 8.324 & 0.004 \\
\hline AA'A & 1.836 & 2.428 & -0.014 & 2.170 & 1.565 & 8.408 & 0.171 \\
\hline$\overline{\mathrm{AB}}$ & 1.806 & 3.520 & \pm 0.0005 & 1.468 & 1.447 & 8.377 & 0.120 \\
\hline$\overline{\mathrm{A}} \mathrm{B} \overline{\mathrm{A}}$ & 1.837 & 2.335 & +0.076 & 2.170 & 1.565 & 8.408 & 0.171 \\
\hline
\end{tabular}

$R_{\mathrm{Si}-\mathrm{C}} \sim 1.8 \AA \mathbb{A}_{g} \sim 2$ эВ, $E_{b} \sim 8.4$ эВ/атом $) \quad[17,24,28,30]$ результатами из литературных источников, что является подтверждением правомерности использованных методов и подходов.

Для оценки зарядовых характеристик структур использован анализ заселенности орбиталей по Малликену [38]. Данный метод позволяет оценить качественное отличие в распределении заряда в материале. При этом для анализа количественных характеристик требуется использование более точных методов расчета заселенности орбиталей.

Объектом исследования являются многослойные структуры на основе атомарно тонкого карбида кремния, отличающиеся взаимным расположением и числом слоев. Каждый слой предложенных структур представляет собой гексагональную сетку, составленную из последовательно чередующихся атомов кремния и углерода.

Рассмотрены следующие 2D аллотропы (представлено строение с не оптимизированной геометрией):

ААА, с одинаковой конфигурацией атомов во всех слоях;

AĀA, с «инверсией» атомов в смежных слоях;

AĀ'A, полученные смещением среднего слоя в $\mathrm{A} \bar{A} \mathrm{~A}$ вдоль одного из векторов трансляции на длину связи $R_{\mathrm{Si-C}}$;

AА'А, полученные смещением среднего слоя в ААА вдоль одного из векторов трансляции на длину связи $R_{\mathrm{Si}-\mathrm{C}}$

AВА, полученные смещением среднего слоя в ААА на вектор $\left[R_{\mathrm{Si-C}} / 2 ; R_{\mathrm{Si}-\mathrm{C}} / 2\right]$;

$\bar{A} \bar{A} \overline{,}$, с «инверсией» атомов среднего слоях относительно АВА.

В качестве модельной структуры для оценки энергетических и геометрических параметров 2D аллотро- пов карбида кремния, выбран графен и мультиграфен, как наиболее исследованные низкоразмерные материалы $[1,2,34-37,39-42]$.

\section{3. Результаты и обсуждение}

По результатам моделирования однослойного $\mathrm{SiC}$ установлено, что длина химической связи $R_{\mathrm{Si-C}}$ составляет $1.80 \AA ̊$, что на $0.38 \AA$ больше длины связи углерод-углерод в графене. Это связано с наличием у кремния более массивного электронного остова, экранирующего ядро, что в итоге выражается в более слабой, чем С-С, связи. Как следствие, наблюдается уменьшение приведенной энергии связи на 2.58 эВ, относительно графена, что для $\mathrm{SiC}$ составляет $E_{b}=8.322$ эВ, указывая на высокую термодинамическую стабильность данной структуры. Между тем данная особенность электронного строения проявляется так же в формировании, наряду с сильной ковалентной связью, слабой ионной, за счет локального перераспределения электронной плотности (Рис. 2). При этом полученная структура является прямозонным полупроводниковым материалом с шириной запрещенной зоны $E_{g} \approx 1.98$ эВ.

Послойный рост политипа АĀA сопровождается осциллирующим изменением удельного атомарного заряда: $1.315 \overline{\mathrm{e}}, 1.273 \overline{\mathrm{e}}$ и $1.329 \overline{\mathrm{e}}$ (ё-элементарный заряд). При этом наблюдается монотонный рост удельной и межслоевой энергии связи. Анализ зарядовых свойств показал формирование положительного не скомпенсированного удельного заряда на поверхности, что можно связать с особенностями морфологии и электронного строения структуры. Так, формирование двухслойной конфигурации сопровождается частичной гибридизацией $p_{z}$-орбиталей атомов в монослоях и образовани- 


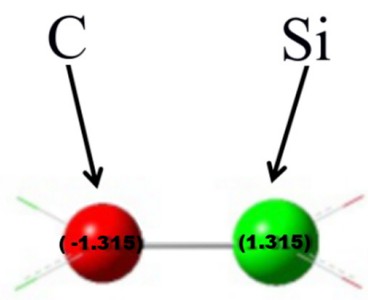

a

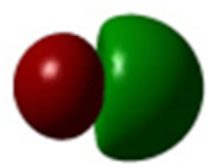

$\mathrm{b}$
Pис. 2. (Color online) Элементарная ячейка однослойного $\mathrm{SiC}$ с обозначением заряда атомов (a), высшие занятые (НОMO) и низшие свободные молекулярные орбитали (LUMO) элементарной ячейки однослойного $\mathrm{SiC}(\mathrm{b})$.

Fig. 2. (Color online) Unit cell of single-layer $\mathrm{SiC}$ witch atomic charge (a), higher occupied (HOMO) and lowest unoccupied molecular orbital (LUMO) in the unit cell of single-layer $\mathrm{SiC}$ (b).

ем дополнительной ковалентной связи между слоями. Перестройка электронной структуры сопровождается снижением удельного атомарного заряда. Так как межслоевая длина связи в такой структуре меньше характерной для $s p^{3}$ гибридизованного состояния объёмного $\mathrm{SiC}$, можно предположить, что степень гибридизации структуры занимает промежуточное значение между $s p^{2}$ и $s p^{3}$. Дальнейший рост такого типа структур приводит к обобществлению частично гибридизованных $p_{z}$-орбиталей среднего слоя, локализации распределенной связи в центре структуры и, как следствие, образованию не скомпенсированного поверхностного заряда и росту межслоевого расстояния.

Похожая эволюция происходит и в структурах типа АВА (Рис. 3). Рассматриваемый аллотроп характеризуется почти вдвое меньшей удельной межслоевой энергией связи и является наиболее стабильной фазой среди остальных политипных модификаций. Анализ параметров структуры показал, что, в данном случае, двухслойная конфигурация образуется за счет гибридизации только половины атомов, благодаря топологической особенности данного политипа, тогда как другая половина атомов располагается в центре гексагонов, образованных атомами соседнего слоя и принимает опосредованное участие в формировании межслоевой связи. Поэтому данный аллотроп характеризуется почти вдвое меньшей удельной межслоевой энергией $E_{b s h}=0.156$ эВ и, соответственно, большими межплоскостными расстояниями, сопоставимыми с длинной связи в объемном $\mathrm{SiC}$. Дальнейший рост структуры так же приводит к обобществлению частично гибридизованных $p_{z}$-орбиталей среднего слоя, преимущественной локализации валентных $p_{z}$-электронов в центреструктуры и формированию положительного удельного заряда на поверхности. При этом, отношение поверхностного заряда в структурах $Q_{b s h-A \bar{A} A} / Q_{b s h-А в A}>2$, что связано c вкладом делокализованных $p_{z}$-орбиталей атомов, расположенных в центрах пентагонов, образованных атомами смежных слоев.

Последовательный рост числа слоев $n$ до 2 и 3 в конфигурации ААА сопровождается трансформацией исходной структуры к типу АВА, и формированием ряда принципиальных особенностей. Так двухслойная конфигурация характеризуется наличием дипольного момента, направленного перпендикулярно плоскости двумерного кристалла. Сравнительный анализ структуры и зарядовых свойств этих двух политипов показал наличие неэквивалентности расположения атомов углерода и кремния. В отличие от АВА, образование ковалентной межслоевой связи в аллотропе AАA происходит за счет общей $p_{z}$-орбитали кремния внутреннего слоя, поэтому, в результате большей электроотрицательности атомов углерода соседних слоёв, внешние слои приобретают слабый отрицательный удельный заряд на поверхности. Кроме того, учитывая идентичность остальных характеристик этих двух типов, можно сделать вывод, что учет зарядовых свойств позволяет определить порядок укладки монослоев.

Послойный рост структуры типа АĀ'A сопровождается ее дестабилизацией относительно однослойного $\mathrm{SiC}$. Для стабилизации упаковки структуры необходима энергия 84 и 14 мэВ/атом. Кроме того, установлено, что поверхностный заряд в структурах данного типа не образуется.

Рост $\bar{A} B \bar{A}$ сопровождается изменением параметров химической связи $\left(R_{\mathrm{Si}-\mathrm{C}}=1.805 \rightarrow 1.843 \rightarrow 1.836 \AA, E_{b}=8.32\right.$ $2 \rightarrow 8.322 \rightarrow 8.408$ эВ/атом) и трансформацией к наиболее энергетически выгодной геометрической конфигурации,
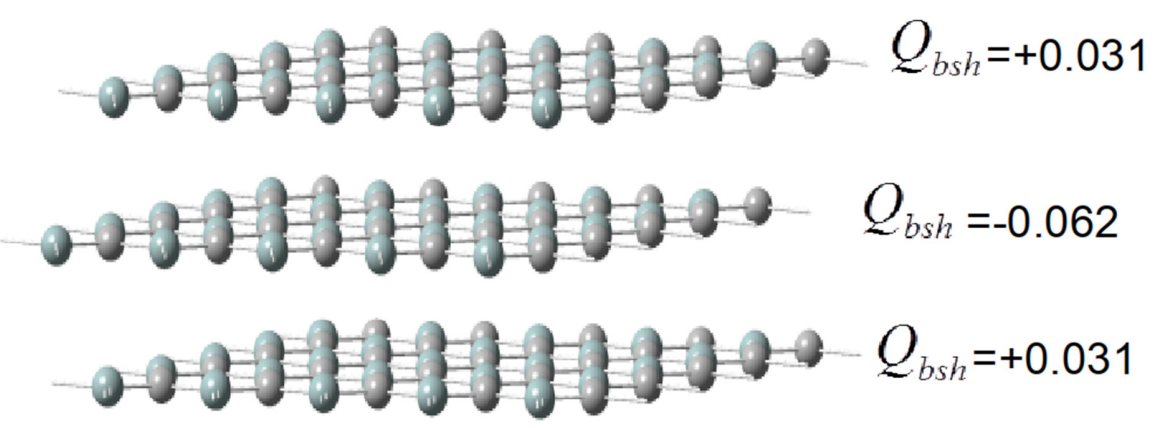

a

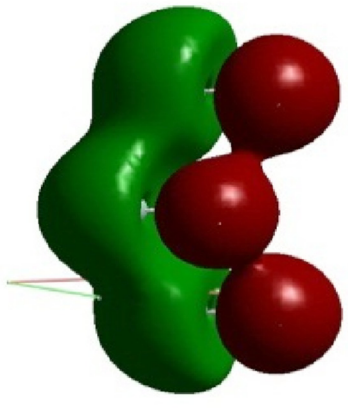

b

Pис. 3. (Color online) Макроячейка $5 \times 5$ структуры АВА с обозначением эффективного удельный поверхностного заряда (а), высшие занятые (HOMO) и низшие свободные молекулярные орбитали (LUMO) элементарной ячейки ABA (b).

Fig. 3. (Color online) Macrocell $5 \times 5$ ABA structure witch the specific charge layer (a), higher occupied (HOMO) and lowest unoccupied molecular orbital (LUMO) in the unit cell of the ABA structure (b). 
соответствующей АĀA политипу, с аналогичной эволюцией зарядовых свойств.

Для структуры АА'А установлено смещение среднего слоя, относительно исходной геометрии, с трансформацией в топологически инвариантный ААА тип. При этом, структура характеризуется максимальным в сравнении с другими межслойным расстоянием $R_{s h} \sim 3.5 \AA$ и является прямозонным полупроводником с шириной запрещенной зоны $E_{g}=1.44$ эВ. Отметим, что характер распределения заряда для трехслойной фазы AA'A аналогичен ААА политипу.

Таким образом анализ величины и знака поверхностного заряда позволяет идентифицировать топологический тип структуры - типа АĀA или ABA и порядок укладки слоев, как в случае ААА и АВА политипов.

\section{4. Заключение и выводы}

При понижении размерности в структурах на основе $\mathrm{SiC}$ установлено формирование отличных от объёмного материала свойств. В частности, при переходе к 2D-аллотропам, при рассмотрении такого явления как политипизм появляется необходимость учёта не только способа укладки слоёв, но и порядка чередования атомов смежных слоёв. При этом, трансляционная симметрия, характерная для объемного $\mathrm{SiC}$ трансформируется и 2D аллатропы имеют слоистую, схожую с мультиграфеном структуру, которая при последовательном увеличении числа слоёв стремится к кристаллической решетке объемного материала. Таким образом, показано, что понижение размерности в 2D кристаллах $\mathrm{SiC}$, с преимущественно ковалентным типом взаимодействия, ведет к появлению новых полиморфных форм, не характерных для объёмного материала.

Так же обнаружено, что послойный рост исследованных структур определяет изменение зарядовых свойств и формирование эффективного поверхностного заряда, а полученные значения эффективного заряда позволяют идентифицировать конкретный структурный тип. Таким образом, учёт перераспределения заряда в процессе роста числа слоев от 1 до 3 для различных аллотропных модификаций $\mathrm{SiC}$ позволяет предположить электрофизический метод сепарации многослойных 2D аллотропов. А особенности зарядовых свойств исследованных структур формируют основу для создания композитных структур на их основе.

Благодарности/Acknowledgements. Работа выполнена при бинансовой поддержке Российского Фонда Фундаментальных Исследований (проект № 16-43-360281 р_а)./ This work was done with the financial support of the Russian Foundation for Basic Research (project № 16-43-360281 p_a).

\section{Литература/References}

1. K.S. Novoselov, A.K. Geim, S. V. Morozov, D. Jiang, Y. Zhang, S. V. Dubonosov, I. V. Grigirieva, A. A. Firsova. Science. 306, 666 (2004). Crossref

2. A. K. Geim. Science. 324, 1530 (2009). Crossref
3. L. Kou, Y. Ma, X. Tau, T. Frauemanheim, A. Du, S. Smith. J. Phys. Chem. C. 119 (12), 6918 (2015). Crossref

4. A.K. Geim, I. V. Grigorieva. Nature. 499, 419 (2013). $\underline{\text { Crossref }}$

5. G. Constantinescu, A. Kuc, T. Heine. Phys. Rev. Lett. 111, 036104 (2013). Crossref

6. J. N. Coleman, M. Lotya, A. O’Neill, S. D. Bergin, P. J. King, U. Khan, K. Young, A. Gaucher, S. De, R. J. Smith. Science. 331, 568 (2011). Crossref

7. Z. Y. Zeng, Z.Y. Yin, X. Huang, H. Li, Q.Y. He, G. Lu, F. Boey, H. Zhang. Angew. Chem. Int. Edit. 50, 11093 (2011). Crossref

8. F. Matusalem et al. Scientific Reports. 7, 15700 (2017). Crossref

9. Z.H. Zhang, W.L. Guo. Phys. Rev. B. 77, 075403 (2008). Crossref

10. P. Vogt, P. Padova, C. Quaresima. Phys. Rev. Lett. 108, 155501 (2012). Crossref

11. H. Sahin, S. Cahangirov, M. Topsakal, E. Bekaroglu, E. Aktrk, R. T. Senger. Phys. Rev. B. 80, 155453 (2009). ㄷossref

12. J. Guan, D. Liu, Z. Zhu, D. Tománek. Nano Letters. 16, 3247 (2016). Crossref

13. I. I. Dolgih, D. V. Avdeev, T. V. Kulikova, L. A. Bityutskaya. KSMF. 20 (2), 305 (2018). (in Russian) [И. И. Долгих, Д. В. Авдеев, Т.В. Куликова, Л. А. Битюцкая. КСМФ, 20 (2), 305 (2018).] Crossref

14. T. V. Kulikova, A. V. Tuchin, A.A. Averin, D. A. Testov, L. A. Bityutskaya, E. N. Bormontov. Journal of Technical Physics. 7, 1025 (2018). (in Russian) [Т. В. Куликова, А. В. Тучин, А. А. Аверин, Д. А. Тестов, Л. А. Битюцкая, Е.Н. Бормонтов. Журнал технической физики. 7, 1025 (2018).] Crossref

15. E. Martínez-Periñán, C.W. Foster, M.P. Down, Y. Zhang, X. Ji, E. Lorenzo, D. Kononovs, A. I. Saprykin, V.N. Yakovlev, G. A. Pozdnyakov, C.E. Bank. Journal of Carbon Research. 3, 20 (2017). Crossref

16. S. Chabi, H. Chang, Y. Xia, Y.Zhu. Nanotechnology. 27, 075602 (2016). Crossref

17. P. Li, R. Zhou, X.C. Zeng. Nanoscale. 6, 11685 (2014). Crossref

18. N. Alaal, V. Loganathan, N. Medhekar, A. Shukla. Phys. Rev. Applied. 7, 064009 (2017). Crossref

19. M.S. Manju, K. M. Ajith, M. C. Valsakumar. Mechanics of Materials. 120, 43 (2018). Crossref

20. F.-Z. Ramadan, H. Ouarrad, L. B. Drissi. J. Phys. Chem. A. 122 (22), 5016 (2018). Crossref

21. S. D. Guo, J.T. Liu. Phys. Chem. Chem. Phys. 20, 22038 (2018). Crossref

22. N. Nouri, G. Rashedi. J. Semicond. 39, 083001 (2018). Crossref

23. P. Miró, M. Audiffreda, T. Heine. Chem. Soc. Rev. 43, 6537 (2014). $\underline{\text { Crossref }}$

24. B. Peng, Y. Zhang, Y. Wang, H. Guo, L. Yuan, R. Jia. Phys. Rev. B. 97, 054401 (2018). $\underline{\text { Crossref }}$

25. G. Gao, N.W. Ashcroft, R. Hoffman. J.Am. Chem. Soc. 135 (31), 11651 (2013). Crossref

26. X. Liu, X. Shao, B. Yang, M. Zhao. Nanoscale. 10, 2108 (2017). Crossref

27. M. Naseri. Physics Letters A. 382 (10), 710 (2018). Crossref 
28. Z. Shi, Z. Zhang, A. Kutana, B. I. Yakobson. ACS Nano. 9 (10), 9802 (2015). Crossref

29. M. Zhao, R. Zhang. Phys. Rev. B. 89, 195427 (2014). Crossref

30. A.L. Falk, P. V. Klimov, V. Ivády, K. Szász, D. J. Christle, W. F. Koehl, Á. Gali, D.D. Awschalom. Phys. Rev. Lett. 114, 247603 (2015). Crossref

31. H. Kraus, V. Soltamov, F. Fuchs, D. Simin, A. Sperlich, P.V. Baranov, G. Astakhov, V. Dyakonov. Scientific Reports. 4, 5303 (2014). Crossref

32. P. Hohenberg, W. Kohn. Phys. Rev. 136, B864 (1964). Crossref

33. W. Kohn, L. Sham. Phys. Rev. 140, A1133 (1965). Crossref

34. A. A. Lebedev, N. V. Agrinskaya, S. P. Lebedev. Technical Physics Letters. 45 (5), 634 (2011). (in Russian) [А. А. Лебедев, Н. В. Агринская, С. П. Лебедев. Письма в ЖТФ. 45 (5), 634 (2011).] Crossref

35. B. Li, P. Ou, Y. Wei, X. Zhang, J. Song. Materials. 11 (5), 726 (2018). Crossref

36. A. Z. Aizahrani, G. P. Srivastava. Braz. J. Phys. 39 (4), 694 (2009). Crossref

37. M. Polini, A. Tomadin, R. Asgari, A. H. MacDonald. Phys. Rev. B. 78, 115426 (2008). Crossref

38. R.S. Mulliken. J. Chem. Phys. 23, 1833 (1955). Crossref

39. E. V. Castro, K.S. Novoselov, S.V. Morozov. Phys. Rev. Lett. 99, 216802 (2007). Crossref

40. Y. Zhang, T. Tang, C. Girit. Nature. 459, 820 (2009). $\underline{\text { Crossref }}$

41. M. F. Craciun, S. Russo, M. Yamamoto. Nature nanotechnology. 4, 383 (2011). Crossref

42. Z. Liu, W. S. Lew, Q. J. Wang. Nanoscale Research Letters. 8 (335), 1 (2013). ․ㅡossref 